\title{
A CRISPR-Cpf1 system for efficient genome editing and transcriptional repression in plants
}

Xu Tang, Levi G. Lowder, Tao Zhang, Aimee A. Malzahn, Xuelian Zheng, Daniel F. Voytas, Zhaohui Zhong, Yiyi Chen, Qiurong Ren, Qian Li, Elida R. Kirkland, Yong Zhang and Yiping Qi

Nature Plants 3, 17018 (2017); published 17 February 2017; corrected 19 June 2017.

In the Supplementary Information file originally published, the bottom oligo sequence in Supplementary Fig. 1b was incorrect. The sequence should have been $3^{\prime}$-ANNNNNNNNNNNNNNNNNNNNNNCCGG-5'. This has now been corrected. 